\title{
DPP controls tracheal cell migration along the dorsoventral body axis of the
}

\section{Drosophila embryo}

\author{
Stéphane Vincent ${ }^{1, *}$, Esther Ruberte ${ }^{1, *}$, Nicole C. Grieder ${ }^{1}$, Chao-Kung Chen ${ }^{2}$, Theo Haerry ${ }^{3}$, \\ Reinhard Schuh ${ }^{2}$ and Markus Affolter ${ }^{1}$ \\ ${ }^{1}$ Abteilung Zellbiologie, Biozentrum, Universtät Basel, Klingelbergstrasse 70, CH-4056 Basel, Switzerland \\ ${ }^{2}$ Abteilung Molekulare Entwicklungsbiologie, Max-Planck-Institut für biophysikalische Chemie, Am Fassberg, D-37077 Göttingen, \\ Germany \\ ${ }^{3}$ Department of Molecular Biology and Biochemistry and Developmental Biology Center, University of California, Irvine, California \\ 92717, USA \\ *These authors contributed equally to this work
}

\section{SUMMARY}

We report that DPP signaling is required for directed tracheal cell migration during Drosophila embryogenesis. The failure of tracheal cells to receive the DPP signal from adjacent dorsal and ventral cells results in the absence of dorsal and ventral migrations. Ectopic DPP signaling can reprogram cells in the center of the placode to adopt a dorsoventral migration behavior. The effects observed in response to ectopic DPP signaling are also observed upon the tracheal-specific expression of a constitutive active DPP type I receptor (TKV $\left.{ }^{2253 D}\right)$, indicating that the DPP signal is received and transmitted in tracheal cells to control their migration behavior. DPP signaling determines localized gene expression patterns in the developing tracheal placode, and is also required for the dorsal expression of the recently identified BRANCHLESS (BNL) guidance molecule, the ligand of the BREATHLESS (BTL) receptor. Thus, DPP plays a dual role during tracheal cell migration. It is required to control the dorsal expression of the BNL ligand; in addition, the DPP signal recruits groups of dorsal and ventral tracheal cells and programs them to migrate in dorsal and ventral directions.

Key words: decapentaplegic, cell signaling, cell migration, transcription factor, Drosophila

\section{INTRODUCTION}

During the development of multicellular animals, a large number of cells originate at considerable distance from their final site they occupy within the organism. To reach this site, these cells have to migrate over other tissues or extracellular substrates receiving and interpreting instructions from their environment which guide them to their target. Such precise cell movements are a prerequisite for the concerted development and the subsequent interconnection of tissues within developing organisms.

Many cell types are motile at some stage during their developmental history, such as cells of the nervous system, muscle cells, primordial germ cells, and endothelial cells forming the blood vessels. Although a coherent picture of how cells generate force and mobility is beginning to emerge (Lauffenburger and Horvitz, 1996; Mitchison and Cramer, 1996), the understanding of how mobility is regulated during development is less advanced. Locally distributed signals dictating the direction of migration are most likely molecules anchored to the membrane of the migration surface, molecules associated with the extracellular environment of the migration path or diffusible factors. Receptors recognizing these guidance molecules as attractive or repulsive must be expressed on the surface of migrating cells and their activation should ultimately lead to the intracellular changes that cause directional migration.

To unravel the mechanisms controlling guided cell movements, genetic approaches have recently been initiated in Caenorhabditis elegans and in Drosophila melanogaster (see Montell, 1994; Howard, 1993; Cuelotti, 1994; Garriga and Stern, 1994). In Drosophila, the developing trachea serves as a model system to study directed cell migration. The tracheal system develops from ten individual small bulges, the tracheal placodes, which form in lateral positions in the trunk segments on either side of the embryo (for detailed descriptions, see Campos-Ortega and Hartenstein, 1985; Hartenstein and Jan, 1992; Manning and Krasnow, 1993; Samakovlis et al., 1996). Upon invagination of the tracheal cells, the complex branching pattern of the tracheal system is established via cell migration, extension and fusion in the absence of further cell division. During this process, defined numbers of cells migrate out from each placode as anterior branches and as ventral and dorsal branches. The establishment of the tracheal network thus requires that groups of cells are instructed with respect to distinct routes which they have to follow. The highly stereotyped development of the tracheal system combined with the powerful genetics of Drosophila should allow a molecular dissection of this process and lead to a better understanding of how cells recognize and interpret migration cues. 
Mutations in a number of genes have been reported to cause abnormal development of the tracheal system. The breathless (btl) gene is strictly required for tracheal cell migration and encodes the Drosophila homolog of the fibroblast growth factor receptor (Glazer and Shilo, 1991; Klämbt et al., 1992). In the absence of $b t l$ function, tracheal cell division as well as general tracheal-cell fate appear to be unaffected, but tracheal cells fail to migrate and, as a consequence, no branches are formed. Interestingly, recent results indicate that the normal activity of BTL in promoting cell migration requires a spatially restricted FGF-like ligand, the product of the branchless $(b n l)$ gene (Sutherland et al., 1996). The dynamic expression pattern of $b n l$ at each position where a new branch will form and grow out, combined with the results obtained in ectopic expression experiments suggest that BNL acts as a chemoattractive guidance molecule (Sutherland et al., 1996; Lee et al., 1996).

In contrast to mutations in the genes $b t l$ and $b n l$, mutations in the two essential DPP receptor genes punt and thick veins $(t k v)$ have been found to affect exclusively the development of certain branches of the tracheal system (Affolter et al., 1994; Ruberte et al., 1995). Here we report that DPP plays a dual role during tracheal cell migration. On the one hand, DPP controls the region-specific activation of $b n l$ in the dorsal part of the embryo. On the other hand, DPP expression dorsal and ventral to the tracheal placode at the onset of migration instructs groups of tracheal cells with respect to their migration behavior. In the absence of DPP signaling, tracheal cells lose their ability to migrate along the dorsoventral body axis. Ectopic DPP signaling in the center of the tracheal placode inhibits anterior migration, and reprograms cells to adopt a dorsoventral migration behavior without interfering with $b n l$ expression. These results suggest that other factors in addition to BNL dictate the direction of migration along the dorsoventral axis; some of these factors might be recognized by tracheal cells only upon the reception of the DPP signal.

\section{MATERIALS AND METHODS}

\section{Drosophila strains}

We used the $t k v^{\text {str-II }}$ (Nüsslein-Volhard et al., 1984), the punt ${ }^{135}$ (Jürgens et al., 1984) and the punt ${ }^{P}$ (Ruberte et al., 1994) alleles to analyze DPP receptor mutant phenotypes. $t v^{\text {str-II }}$ represents a null allele, whereas punt ${ }^{135}$ and punt $^{P}$ behave as hypomorphic alleles. The weaker ventral tracheal phenotype observed in punt as compared to $t k v$ is most likely due to the longer maternal perdurance of the punt product. To generate the 1-eve-1, punt recombinant chromosome, the punt $^{P}$ allele was used. $h s G A L 4$ and UASdpp transgenic fly strains were described by Ruberte et al. (1995); UASpunt, UAStkv and $U A S t k v^{Q 253 D}$ were described by Nellen et al. (1996), and kindly provided by K. Basler and D. Nellen prior to publication. The 1-eve1 strain is described by Perrimon et al. (1991). Construction of dominant negative sax was as follows. A fragment that encodes a constitutive active SAX protein, SAXQ263D, was cloned as a XhoI$X b a \mathrm{I}$ fragment into the same sites in pUAST. The plasmid was cut with NheI and SpeI and ligated with an oligonucleotide spacer that contains stop codons in all frames and an internal SpeI site for identification. This deletion removes the whole kinase domain and creates a protein of 261 amino acids. Transformants with single, double and multiple insertion sites were obtained by injection. The line used in this study contains several inserts on the second and third chromosome. Fly strains containing btlGAL4 constructs on the X, the second or the third chromosome were kindly provided by Shigeo Hayashi
(Shiga et al., 1996). The btl promoter drives GAL4 expression in the tracheal placode to high levels from late stage 10 on; no detectable expression is observed in the cells surrounding the placode. However, significant levels of GAL4 are also produced in the anterior and posterior midgut primordium, leading to the induction of labial in the endoderm upon the expression of $d p p$ or $t k^{Q 253 D}$. We also used a fly strain containing a btlGAL4 construct on the second chromosome and a NLS-GFP-lac Z fusion construct on the third chromosome (Shiga et al., 1996); this allows the induction of GAL4 in the tracheal system and tracheal development to be followed with the nuclear $\beta$ galactosidase.

\section{Whole-mount in situ hybridizations}

When DNA probes were used, in situ hybridization to whole-mount embryos was performed as described by Tautz and Pfeifle (1989) with minor modifications. When RNA probes were used, in situ hybridization was done according to Hauptmann and Gerster (1994), but without proteinase $\mathrm{K}$ digestion. In the experiments in which we detected RNA and protein, the primary antibody was incubated (after hybridization) with the anti-DIG antibody, and the secondary antibody was added after the staining reaction for the RNA probe. Probes used were derived from cDNAs of bnl (Sutherland et al., 1996; kindly obtained from D. Sutherland and M. Krasnow), kni (Nauber et al., 1988), and $d p p$ (St. Johnson et al., 1990).

\section{Immunostainings and microscopy}

Embryos were immunostained according to standard procedures (Ashburner, 1989), followed by the addition of a secondary antibody conjugated with biotin or with alkaline phosphatase. The distribution of the secondary antibody was revealed either by using horseradish peroxidase $\mathrm{ABC}$ kit (Vectastain) or by staining for alkaline phosphatase activity. A rabbit LAB antiserum was prepared as described by Chouinard et al. (1991), and purified (U. Nussbaumer and M. A., unpublished). The SAL antibody was described previously (Kühnlein et al., 1994). Anti-tracheal lumen mAb 2 A12 were obtained from C.S. Goodman and N. Patel. To visualize $\beta$-galactosidase expression, a monoclonal antibody (Promega) was used. In all cases, mutant embryos were unambiguously identified using marked balancer chromosomes or previously described mutant phenotypes. For light microscopy, immunostained embryos were viewed in a Zeiss Axiophot compound microscope using differential interference contrast optics. For documentation, images were photographed on EKTAR 100 (Kodak) film.

\section{Heat-shock experiments}

Embryos were collected overnight on grape-juice plates, submitted to a heat-treatment at $38^{\circ} \mathrm{C}$ for 20 minutes, and allowed to continue development for an additonal four hours at $25^{\circ} \mathrm{C}$. Embryos were then fixed and analyzed. To identify those embryos that contained both the hsGAL4 and the UASdpp or UAStkv ${ }^{Q 253 D}$ constructs, respectively, we used the purified polyclonal LAB antiserum. LAB protein is readily induced in the midgut endoderm upon ubiquitous DPP signaling (Ruberte et al., 1995). As already noted for LAB induction, the effects of ectopic DPP on tracheal development depended entirely on the presence of both the punt and the $t k v$ gene products (see Ruberte et al., 1995); no redirection of cell migration upon ectopic DPP expression was observed in $t k v$ mutants. When embryos were analyzed 4 hours after heat treatment, only stage 12 and 13 embryos which had previously received ubiquitous, high levels of DPP showed tracheal cell migration defects. Stage 14 embryos in which DPP had been expressed ubiquitously 4 hours earlier, in late stage 11 (monitored by the extended LAB expression in the endoderm), showed only minor migration defects although they just completed the predominant tracheal cell migration period. This demonstrates that DPP induction after stage 11/early stage 12 had no effect on branch formation although high levels of DPP were present throughout the major migration phase. These observations were confirmed by col- 
lecting staged embryos (+/- 30 minutes). Embryos that received ubiquitous DPP prior to stage 11 showed a dorsalization of the epidermis and an enlargement of the tracheal placode along the anterior-posterior axis; no such dorsalization was observed when DPP was induced at stage 11 or later stages; i.e. alterations in tracheal cell migrations occurred in the absence of a dorsalization of the epidermis.

\section{RESULTS}

\section{The two DPP receptors TKV and PUNT are required for tracheal development}

Embryonic lethal mutations in the genes punt and $t k v$ were first identified by Nüsslein-Volhard, Wieschaus and collegues by the defects in closure of the dorsal epidermis in such mutants (Nüsslein-Volhard et al., 1984; Jürgens et al., 1984). Subsequent analysis of punt and $t k v$ mutant embryos revealed that they display a number of additional common defects in a variety of tissues (Affolter et al., 1994; Brummel et al., 1994; Nellen et al., 1994; Penton et al., 1994). In particular, both mutations lead to distinct defects in the developing tracheal system in the embryonic trunk region (Affolter et al., 1994; Ruberte et al., 1995). In $t k v$ mutants, the branches of the developing tracheal system which grow out in a dorsal direction (dorsal branches; see schematic representation for nomenclature used) are absent, and the branches which grow out ventrally (ganglionic branches, lateral trunk) are severely affected and do not form properly (Fig. 1D). The two major branches which grow towards anterior (the dorsal trunk anterior and the visceral branches), are largely unaffected and develop normally during later stages of embryogenesis. In punt mutant embryos, a similar phenotype is observed; no dorsal branches develop and malformation of ventral branches is also seen occasionally (Fig. 1C). Consistent with the observation that the available punt alleles are thermosensitive and most likely hypomorphs (Letsou et al., 1995), the frequency of these ventral defects is increased at $29^{\circ} \mathrm{C}$ (data not shown; see Materials and Methods). Thus, punt and tkv activities are required for the establishment of the dorsal and ventral branches of the developing trachea. Consistent with the genetic requirement of punt and $t k v$ for the proper development of the tracheal system, both genes are transcribed in the tracheal placode. punt is expressed ubiquitously throughout embryogenesis (Ruberte et al., 1995), while $t k v$ transcription is turned on strongly in the placode just prior to the invagination of the latter and the initiation of tracheal cell migration (Affolter et al., 1994).

\section{DPP is expressed on the dorsal and ventral side of the invaginating tracheal placode}

It has previously been shown that the TKV and PUNT transmembrane serine-threonine kinase receptors are required in concert to transduce the DPP signal, and that their functions cannot be replaced by other known type I or type II receptors (Ruberte et al., 1995). In addition, the temporal and spatial specificity with which DPP determines embryonic cell fates appears to be controlled primarily by the restricted availability of the DPP ligand (Ruberte et al., 1995). To gain insight into how the DPP ligand might regulate tracheal cell migration through TKV and PUNT, we analyzed the spatial distribution of DPP with respect to the developing tracheal primordia.
During the slow phase of germ band extension when tracheal cells can first be identified using available markers, DPP is expressed in a large band covering the entire dorsal ectoderm (Fig. 2A,B). Somewhat later, but still prior to the invagination of the tracheal primordia, DPP is expressed in a segmentally repeated pattern in the lateral ectoderm aligned with the ventral margin of the tracheal pits (Fig. 2D). Dorsal to the tracheal placode, DPP is expressed along the entire anteroposterior axis in the dorsal-most ectodermal cell. No DPP expression is seen in tracheal cells of the placode and between the dorsal and lateral expression domains. Thus, just prior to the initiation of tracheal cell migration (Fig. 2E), DPP is expressed in ectodermal cells located dorsally and ventrally of the tracheal placode. Therefore, the lack of the dorsal and ventral branches in $t k v$ mutant embryos could result from a failure of the dorsal- and ventral-most tracheal cells to respond to the localized source of DPP, and this failure to transmit the DPP signal could subsequently result in the inability to initiate cell migration along the dorsoventral axis.

The most direct test for this hypothesis would be to examine $d p p$ mutant embryos for possible tracheal defects. However, null mutations in $d p p$ lead to a strong ventralization of the embryo which results in the complete lack of the tracheal anlagen. In addition, none of the numerous $d p p$ alleles has been shown to affect specifically the expression domains around the tracheal placode. Therefore, due to the lack of such $d p p$ lossof-function alleles, we have first focused our studies on artificially generated gain-of-function experiments which allow tracheal defects brought about by ectopic activation of the DPP signaling pathway to be examined.

\section{Ectopic expression of DPP leads to altered tracheal cell migrations}

To test for a possible involvement of DPP in the control of tracheal cell migration, we expressed DPP ubiquitously under the indirect control of a heat-shock promoter and analyzed the migration behavior of tracheal cells four hours later using antibodies outlining the tracheal tree. Ubiquitous DPP induction at the extended germ stage, prior to the onset of tracheal cell migration, leads to striking developmental alterations: tracheal cells failed to migrate anteriorly resulting in the total absence of the dorsal trunk and the visceral branches. Instead, all cells remained associated with those migrating along the dorsoventral body axis (Fig. 3B). Embryos which received the heatshock somewhat later, and therefore expressed ubiquitous DPP during the active migration phase but only after placode invagination and initiation of migration, did not show any defects in cell migration (see Materials and Methods). This suggests that DPP acts early at the placode stage to respecify cells towards an altered migration behavior. Indeed, the expression of DPP in the developing placode (see below) causes the same change in the migration behavior (Fig. 3C,D). Examination of older embryos that continuously express DPP in tracheal cells from the placode stage on showed that not only was anterior migration completely blocked but additionally, supernumerary cells migrated towards the dorsal side of the embryo and accumulated along the dorsal midline (compare Fig. 3F with 3E).

Interestingly, the tracheal defects observed under conditions of ectopic and ubiquitous DPP expression are reciprocal to the alterations found in the DPP receptor mutants: whereas lack of 

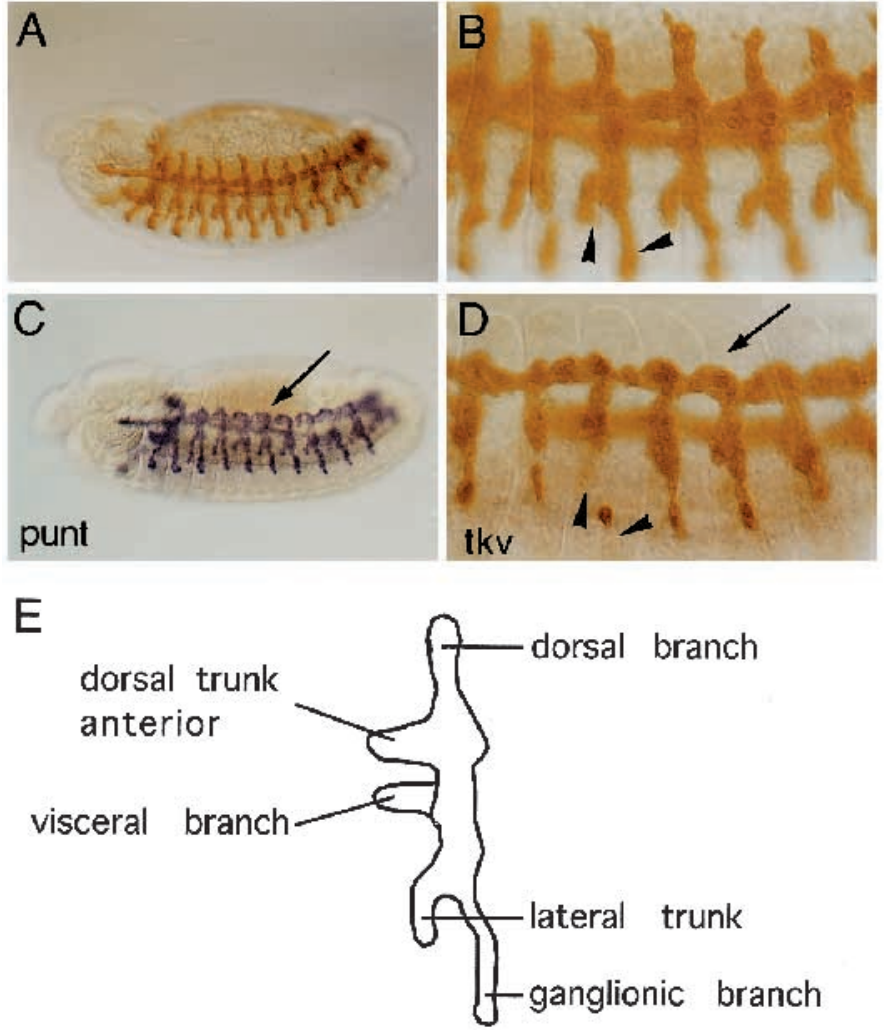

Fig. 1. Tracheal cell migration defects in the DPP receptor mutants tkv and punt. The tracheal system of stage 14 embryos is followed with the aid of the 1-eve-1 chromosome in wild-type (A,B), punt (C) and $t k v(\mathrm{D})$ mutant embryos. In punt mutants, all dorsal branches are absent, and defects in the ventral branches are occasionally observed when embryos are collected at $29^{\circ} \mathrm{C}$. Dorsal branches (arrow) are lacking in $t k v$ mutants, and ganglionic branches and the lateral trunk are severely affected (arrowheads). E shows a schematic representation of a typical tracheal metamere of a stage 14 embryo. The nomenclature used is adopted from Manning and Krasnow (1993) with minor modifications.

DPP signaling results in a failure of tracheal cells to migrate along the dorsoventral axis without significantly affecting anterior migrations, ubiquitous DPP signaling suppresses anterior migrations without interfering with dorsoventral migration. These observations support the interpretation that in wild-type embryos, dorsal and ventral tracheal cells which receive the DPP signal respond to it by migrating along the dorsoventral body axis, and that those cells that do not receive the DPP signal adopt an anterior migration behavior.

\section{DPP and its receptors act in tracheal cells}

Altered cell migration in the absence of DPP signaling or under ectopic DPP signaling could either be due to changes occurring in tracheal cells themselves, to changes occurring in the environment of the tracheal placode, or to a combination of both. To test for a tracheal cell-specific requirement for DPP signaling, we expressed a constitutively active form of the TKV receptor (referred to as TKVQ253D; Nellen et al., 1996) exclusively in tracheal cells, using a tracheal-specific GAL4 line (btlGAL4; see Shiga et al., 1996 and Materials and Methods). The alterations in migration behavior we observed
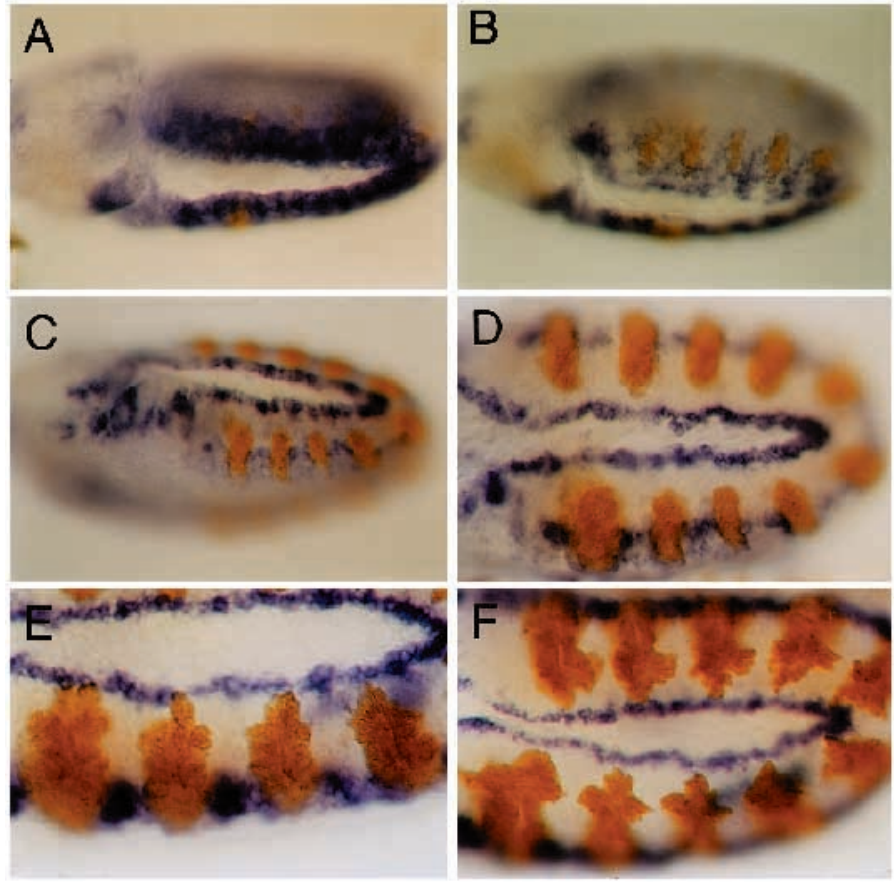

Fig. 2. $d p p$ is expressed in cells apposing the dorsal and ventral sides of the tracheal placode. The tracheal placode is stained brown with the tracheal marker 1-eve-1; $d p p$ RNA is revealed in blue using a DIG-labeled RNA antisense probe. (A) Tracheal cells are already detectable during germ band extension when $d p p$ is still expressed in the entire dorsal ectoderm. (B) $d p p$ expression in this domain subsequently fades, and is reinitiated in the dorsal-most cell and in a lateral, punctuated stripe (C). Before and after the invagination of the tracheal placode ( $\mathrm{D}$ and $\mathrm{E}$, respectively), $d p p$ expression is detected dorsal and ventral to the placode in ectodermal cells. In F, tracheal cells have initiated migration.

were very similar to those seen after ubiquitous DPP expression, i.e. no dorsal trunk developed, and numerous cells migrated towards the dorsal side (Fig. 3G,H). Since TKVQ253D acts in a cell-autonomous manner, and its localized expression in tracheal cells alters their migration, we conclude that the activation of the DPP signal transduction pathway in tracheal cells at the placode stage programs these cells to migrate along the dorsoventral body axis.

While these experiments confirm that the change in migration in the presence of ectopic DPP signaling is a (tracheal) cell autonomous effect, they do not determine whether the loss of DPP signaling, which leads to a failure to migrate dorsally and ventrally in $t k v$ (and punt) mutant embryo, is due to a loss of DPP reception in tracheal cells. Since neither $t k v$ nor punt mutant alleles are available that specifically remove the expression of these DPP receptors in the developing tracheal system, we expressed a dominant negative version of the DPP type I receptor saxophone (Brummel et al., 1994; Nellen et al., 1994; Penton et al., 1994; Singer et al., 1997) in the developing tracheal system and looked for the loss of dorsal and ventral branches (i.e. resembling a $t k v$ mutant phenotype). We have consistently observed the loss of dorsal and ventral branches upon the expression of the truncated version of the SAXOPHONE receptor in wild-type embryos (Fig. 3I,J). The expression of a similar dominant negative form of the TKV 
receptor resulted in the same phenotypes, but at a lower penetrance (data not shown).

From these experiments, we conclude that the activation of the DPP receptors in tracheal cells is necessary for their proper migration behavior. Consistent with this interpretation, and as presented in the next section, we find that DPP signaling controls gene expression in the tracheal placode in a manner that reflects the different migration behaviors observed.

\section{DPP signaling determines localized gene expression patterns in the tracheal system}

To characterize in more detail the alterations induced in tracheal cells after loss of DPP signaling or after ubiquitous DPP signaling, we analyzed, (1) the expression of a gene specifically activated in the tracheal system in the dorsal trunk, i.e. in a structure which is lost upon ectopic DPP expression, and (2) the expression of a gene activated in the dorsal and gan- glionic branches, i.e. structures lost in the absence of DPP signaling.

spalt (sal) is strongly expressed in dorsal trunk cells in stage 14 embryos and is necessary for the directed anterior migration of these cells (Kühnlein et al., 1994; Kühnlein and Schuh, 1996; Fig. 4A). SAL is expressed in the dorsal trunk in punt and $t k v$ mutant embryos (Fig. 4C). However, embryos in which the DPP signaling pathway has been activated in all tracheal cells at the placode stage fail to accumulate SAL (Fig. 4E). This lack of SAL expression correlates with the absence of the dorsal trunk upon ectopic DPP signaling.

In contrast to sal, the gene knirps (kni) is activated in the developing tracheal system in all the branches (dorsal and ganglionic branch, lateral trunk anterior) which we propose to develop under the control of DPP (Fig. 4B; Chen, Kühnlein, Eulenberg, Vincent, Affolter and Schuh, unpublished). Interestingly, and consistent with this proposal, kni expression is
Fig. 3. Tracheal cell migration can be altered by ectopic activation of the DPP signal transduction pathway. Wholemount embryos show the development of the tracheal system using the 1-eve-1 marker chromosome (A-C,E,F) or a nuclear GFP- $\beta$-galactosidase fusion protein $(D, G, H)$ both detected with anti- $\beta$-galactosidase antibodies and shown in brown. In the embryo shown in $\mathrm{B}, d p p$ was ubiquitously expressed under the indirect control of a heatshock promoter, and the tracheal system analyzed 4 hours after DPP induction. In the embryos shown in $\mathrm{C}, \mathrm{D}$ and $\mathrm{F}, d p p$ was expressed in tracheal cells exclusively, with the aid of a btlGAL4 transgene (see Materials and Methods). In the embryos shown in H, DPP signaling was activated in tracheal cells by the expression of the UAStkvQ253D construct with the driver line btlGAL4. Upon ubiquitous expression of DPP during stage 11, the subsequent development (B) of all anteriorly growing branches (dorsal trunk, arrowhead; visceral branch, arrow) was inhibited (compare $\mathrm{B}$ to the wild-type embryo in A). The same phenotype was observed when $d p p$ was expressed in the developing tracheal placode (C,D). In stage 17 embryos, many cells moved to the dorsal side of the embryo upon continuous $d p p$ expression in the developing tracheal system (arrow in $\mathrm{F}$, compare to E). The dorsal branches consist of 5 to 7 cells in wild-type embryos (E,G; Samakovlis et al., 1996); upon ectopic DPP signaling, up to 25 cells can be integrated into the dorsal branch (F,H and data not shown). The effects observed upon the expression of $d p p$ in the developing tracheal system were similar to the effects observed upon the expression of the $U A S t k v^{Q 253 D}$ construct under the control of btlGAL4 (compare $\mathrm{H}$ to $\mathrm{B}, \mathrm{C}, \mathrm{D})$. Expression of a dominant negative version of the DPP type I receptor SAX in the tracheal system leads to $t k v$ like phenotypes $(\mathrm{I}, \mathrm{J})$. Tracheal development was analyzed using the expression of dominant negative UASsax constructs under the indirect control of btlGALA. The outline of the tracheal system was revealed using antibody $2 \mathrm{~A} 12$. I shows a ventral view, J a dorsal view of a stage 15/16 embryo.

Expression of the dominant negative SAX receptor leads to the occasional absence of ganglionic branches (arrowheads in I) or dorsal branches (arrowheads in $\mathrm{J}$ ), reminiscent of $t k v$ or punt mutant phenotypes. In addition, misdirected dorsal branches which cross the segment boundaries were observed at a high frequency (see J). However, this phenotype was also observed upon the expression of other gene products in the developing tracheal system, and is therefore difficult to interpret. See also Fig. 6 for additional illustrations of the tracheal phenotype upon the expression of the dominant negative SAX receptor.
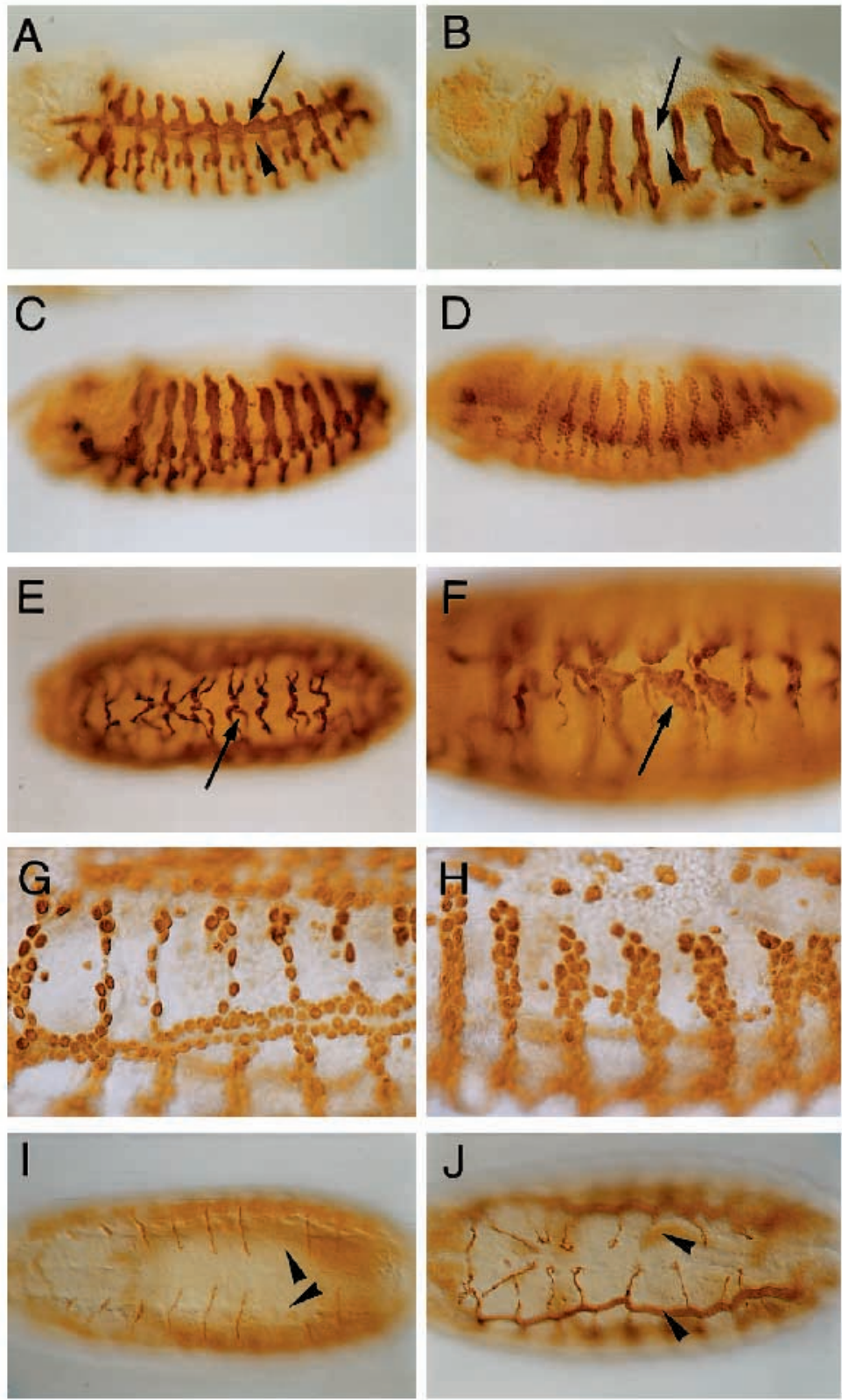
Fig. 4. DPP regulates the expression of genes in subsets of the tracheal cell population. The expression of $s a l$ (A,C,E) was analyzed with a SAL specific antiserum, the expression of kni $(\mathrm{B}, \mathrm{D}, \mathrm{F})$ with a kni antisense RNA probe. Embryos shown are wild-type $(\mathrm{A}, \mathrm{B})$ or $t k^{\text {str-II }}$ (C,D). The embryos shown in $\mathrm{E}$ and $\mathrm{F}$ express the constitutive active TKVQ253D receptor under the indirect control of the $b t l$ enhancer (see Materials and Methods). sal expression is normal in the absence of the zygotic function of $t k v(\mathrm{C})$, but absent from the tracheal system of embryos in which DPP signaling is constitutively activated in tracheal cells (E). In wild type embryos, kni is expressed in the dorsal branch, the ganglionic branch, the lateral trunk and the visceral branch (B). In $t k v$ mutant embryos, kni expression is completely lost in all dorsal and ventral tracheal cells, but still detectable in the visceral branches (D). All tracheal cells express high levels of kni upon the activation of the DPP signaling pathway $(\mathrm{F})$.
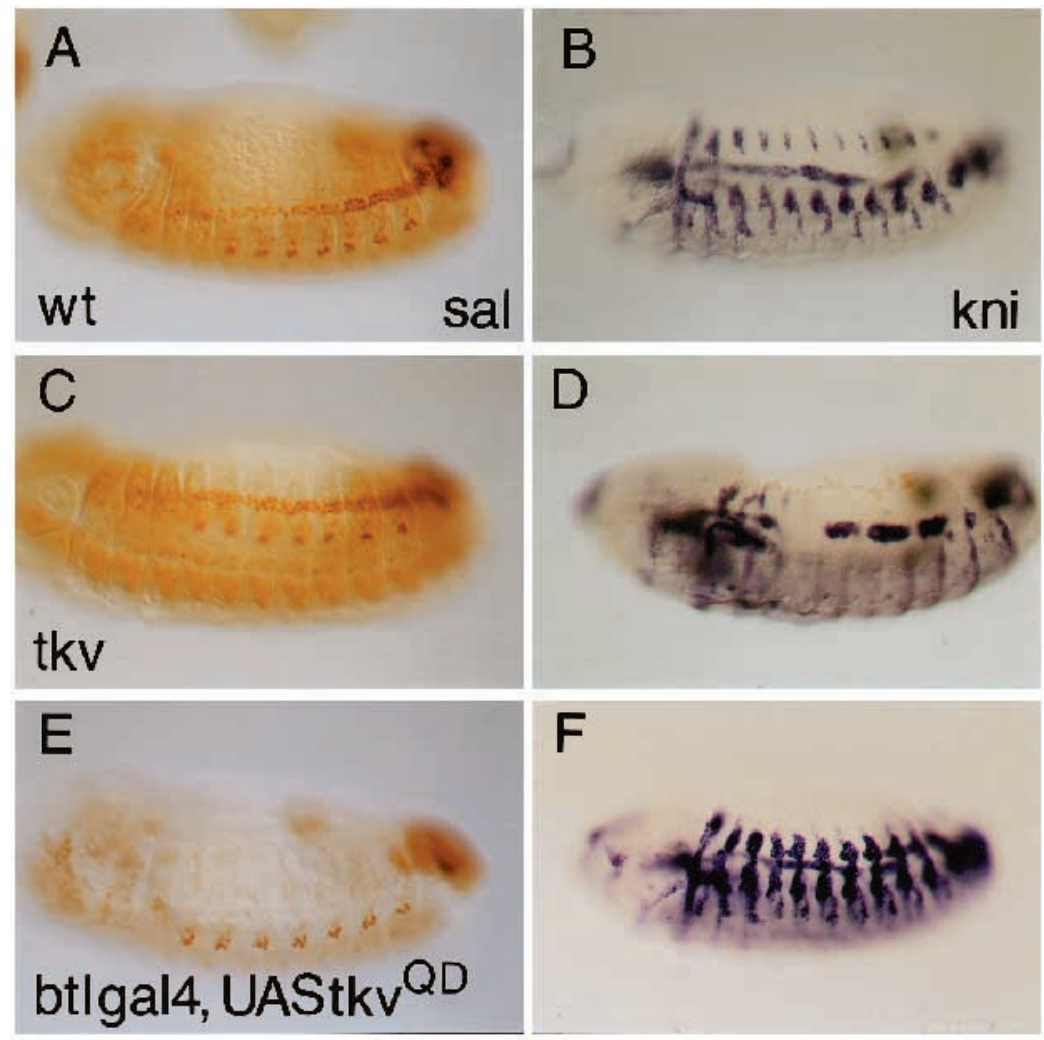

lost in $t k v$ mutants (Fig. 4D; kni expression only persists in the visceral branches of $t k v$ mutants). More strikingly, $k n i$ expression is turned on in all tracheal cells after constitutive DPP signaling (Fig. 4F). Thus, $k n i$ represents a DPP target gene in tracheal cells (see also Discussion).

These results suggest that the phenotypes observed in the loss- and gain-of-function situations are due to changes in cell identities within the tracheal system, rather than to nonspecific inhibition of certain migration behaviors.

\section{DPP is also required for the correct expression of the BNL ligand}

To test whether directed migration requires the activation of the DPP signaling pathway in tracheal cells exclusively, we tried to rescue the $t k v$ and punt tracheal defects by the expression of UAStkv and UASpunt transgenes, respectively, using the tracheal-specific btlGAL4 driver line. A rescue should in principle be possible since the DPP ligand is still expressed around the placode in $t k v$ and punt mutants (Affolter et al., 1994). However, in both rescue situations, we never observed the migration of tracheal cells towards the dorsal side of the embryo, and using lumen-specific markers, we never observed the formation of dorsal branches (data not shown). In addition, the expression of the constitutively active TKVQ253D receptor under the indirect control of btlGAL4 does not cause the formation of dorsal branches in $t k v$ mutants despite its ability to disrupt anterior migration (data not shown; see Discussion).

The failure to rescue dorsal branch defects of $t k v$ and punt mutant embryos by the tracheal-specific expression of $t k v$, $t k v^{Q 253 D}$ or punt cDNAs suggests that DPP signaling might also be required for the establishment of migration cues in the envi-
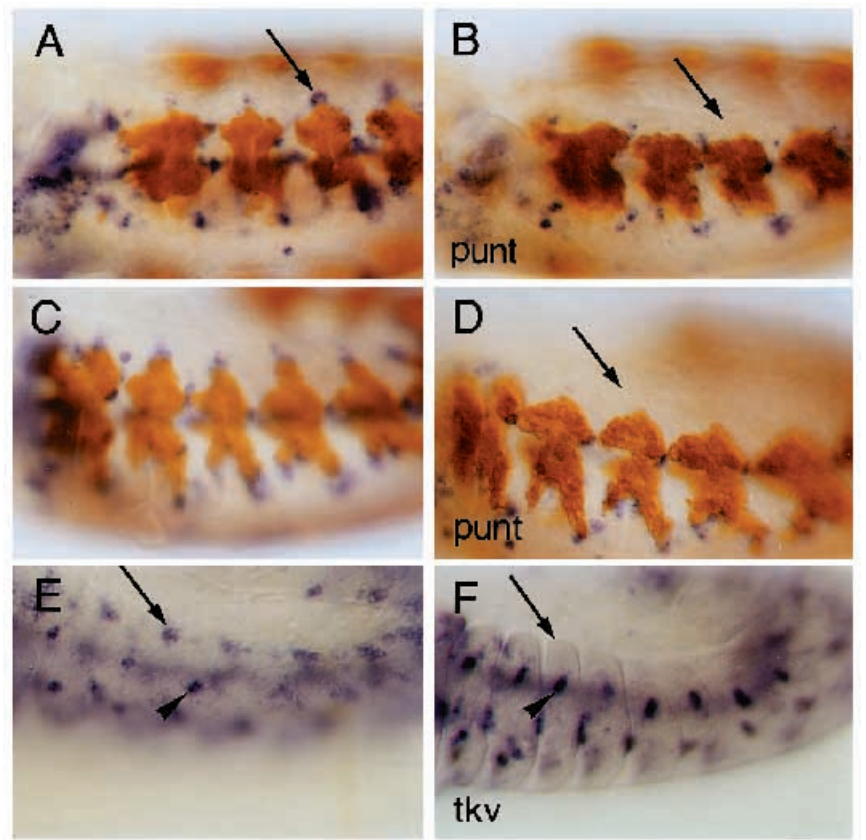

Fig. 5. $b n l$ expression is altered in $t k v$ and punt mutants. Embryos shown are wild type (A,C,E), homozygous for $\operatorname{punt}^{P}(\mathrm{~B}, \mathrm{D})$ or $t k v^{\text {str-II }}$ (F). To visualize the tracheal cells in the punt mutant background, we used a punt, 1-eve-1 recombinant chromosome (see Materials and Methods). In stage 11 punt mutant embryos, the dorsal spots of $b n l$ expression are dramatically reduced or virtually absent (arrow in B) when compared to wild type (arrow in A). During germ band retraction, dorsal expression of $b n l$ is strongly reduced in punt mutants (arrow in D) and not detectable in $t k v$ mutants (arrow in F). Lateral expression of $b n l$ (arrowhead in $\mathrm{E}$ and $\mathrm{F}$ ) is not affected in $t k v$ mutants $(\mathrm{F})$. 
ronment of the tracheal placode. BNL-dependent activation of the BTL receptor is essential for tracheal cells to migrate, and BNL is displaced in patches around the developing tracheal placode (Sutherland et al., 1996). Therefore, we examined $b n l$ expression in $t k v$ and punt mutants. As shown in Fig. 5, bnl transcription is indeed affected in both DPP receptor mutants. The dorsal-most patches of $b n l$ expression which prefigure the formation of the dorsal branches are severely reduced in punt mutant embryos (Fig. 5B,D, compare with A,C) and absent in $t k v$ mutants (Fig. 5F). Therefore, and in addition to its trachealspecific function (see above), DPP signaling is also essential for the proper spatial expression of the BNL ligand on the dorsal side of the embryo.

\section{DPP signaling can alter cell migration without affecting BNL expression}

The observed changes in cell migration upon the expression of constitutively active and dominant negative DPP receptors in a tracheal-cell-specific manner suggests that DPP signaling can alter migration without affecting the expression of the guidance molecule BNL. To confirm this, we have analyzed $b n l$ expression in both situations. As shown in Fig. 3, dorsal branch formation is partially inhibited when DPP signaling is lowered in the developing tracheal system by the expression of a dominant negatively acting SAX receptor. This loss of dorsal branches after the downregulation of the DPP signaling pathway occurred without a concomitant change in the expression pattern of $b n l$ (Fig. 6A,B). The same observation was made upon ectopic activation of the DPP signaling pathway; tracheal cells did not migrate anteriorly despite the presence of BNL in the appropriate position between the tracheal metameres (Fig. 6C). These results confirm that DPP mediated changes in tracheal cell migration occurred independently of $b n l$ expression. Therefore, $b n l$ is not sufficient to

Fig. 6. DPP can alter cell migration without affecting $b n l$ expression. In $\mathrm{A}$ and $\mathrm{B}, b n l$ expression is shown in embryos in which a dominant negative version of the SAX DPP receptor has been expressed under the indirect control of the $b t l$ enhancer (see Materials and Methods). A dorsal branch did not form (arrow) despite the presence of the dorsal spot of $b n l$ expression (arrowhead). In C, bnl expression is shown in an embryo in which the dominant active TKVQ253D receptor has been expressed in the tracheal placode. Cells did not migrate anteriorly (arrow) despite the presence of $b n l$ expression in the appropriate position (arrowhead). direct outgrows of tracheal branches, and tracheal cells need additional signaling input to respond to the BNL ligand in an appropriate manner.

\section{DISCUSSION}

\section{DPP controls dorsoventral cell migration in the Drosophila embryo}

We have previously suggested that the close apposition of DPP secreting cells dorsally and ventrally to the invaginating tracheal placode locally activates the DPP receptor complex in dorsal and ventral tracheal cells and recruits them for migration along the dorsoventral body axis (Affolter et al., 1994). This suggestion was based on the tracheal phenotypes of $t k v$ and punt loss-of-function mutants, and on the expression patterns of the DPP signaling molecule and its receptors (Affolter et al., 1994; Ruberte et al., 1995).

We have now shown that DPP signaling is indeed required in tracheal cells for their subsequent migration along the dorsoventral body axis. Most strikingly, ectopic DPP signaling can reprogram tracheal cells with the consequence that supernumerary cells migrate towards dorsal positions (Fig. 3). DPP

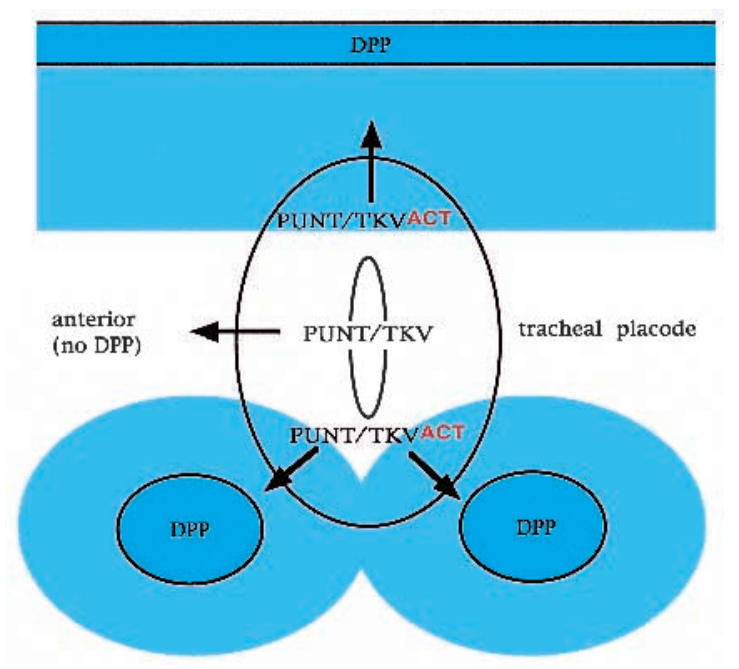

Fig. 7. Tracheal cell migration is controlled by the local secretion of DPP. During the invagination of the tracheal placode and prior to the onset of tracheal cell migration, the $d p p$ gene is transcribed along the entire anteroposterior axis in the dorsal-most cell, and in lateral spots anterior and posterior to the ventral limits of the placode (shown in deep blue). Only ventral and dorsal tracheal cells are reached by the secreted DPP molecule in the limited time span during which tracheal cells respond to DPP with regard to their migration behavior. The transmission of the DPP signal (schematically depicted as PUNT/TKV ${ }^{\text {ACT }}$ ) allows dorsal and ventral cells to migrate dorsally and ventrally, respectively, directed by BNL and possible other factors (see Discussion). Note that the arrows do not indicate a chemoattractive role of DPP, but indicate the migration direction of groups of cells. The determination of dorsal (and possibly ventral) tracheal cells is likely to occur after placode invagination. As the cells invaginate, they maintain an epithelial organization continuous with epithelial cells at the surface; therefore, the dorsal-most cells before invagination are not identical to the dorsal-most cells after the invagination process. These latter cells require the DPP signal and induce $k n i$ immediately after invagination (data not shown). 
acts early, before or during the initiation of cell migration, since the ubiquitous activation of the DPP signaling pathway during the actual migration phase has no effect on the direction of migration. Thus, DPP does not play a chemoattractive role but appears to regionalize the tracheal placode selecting groups of cells and programing them to adopt defined migration behaviors during subsequent development (see Fig. 7). The altered expression patterns of SAL and kni support this interpretation. sal function, for example, is required for anterior migration of dorsal trunk cells (Kühnlein and Schuh, 1996). The suppression of sal by ectopic DPP signaling presumably causes the failure of putative dorsal trunk cells to migrate towards the anterior. However, it is important to note that ectopic DPP signaling does not simply mimic the sal phenotype. Many cells which have received the ectopic DPP signal move dorsally (Fig. 3); in sal mutant embryos, no increase in the number of cells moving dorsally is observed, but more cells are recruited to (or at least associated with) the transverse connective and the spiracular branches (Kühnlein and Schuh, 1996; unpublished observation).

Although it has not been possible to demonstrate directly that the localized expression of DPP around the invaginating placode is the relevant DPP source controlling tracheal cell migration (DPP is expressed more broadly in the dorsal ectoderm prior to placode formation), several observations are in line with this interpretation: (1) in $t k v$ mutants, defects are only visible in dorsally and ventrally migrating cells; (2) DPP signaling in the entire placode affects cells in the center, those that would normally migrate towards anterior in the lateral region of the embryos. These observations lead us to argue that DPP signaling is only initiated in (and essential for) dorsal and ventral tracheal cells (with respect to their migration behavior). This strongly suggests that the dorsal and ventral sources of DPP neighboring the tracheal placode, after its invagination, control tracheal cell migrations.

A number of observations support the second prerequisite of our model, namely that DPP controlled migration behaviors require the activation of the DPP signaling complex in tracheal cells. (1) Tracheal cell migration can be respecified by the tracheal-specific expression of a constitutively active TKV receptor. (2) Gene expression patterns in tracheal cells change in both loss of and ectopic DPP signaling conditions, strongly arguing that the DPP signal is received by the tracheal cells themselves. (3) The expression of dominant negative forms of the known DPP type I receptors in the tracheal primordium induces phenotypes strongly resembling those observed in the tracheal system of $t k v$ (and punt) mutants (absence of dorsal and ventral branches). (4) Expression of a UAStkv construct under the indirect control of btlGALA in $t k v$ mutants resulted in the rescue of kni expression in dorsal and ventral tracheal cells, despite the failure to rescue the dorsal migration phenotype (data not shown). These results demonstrate that the DPP receptors are needed in dorsal and ventral tracheal cells for $k n i$ transcription and for proper cell migration.

\section{TKV and PUNT are required for bnl expression}

It has recently been shown that a FGF signaling pathway including $b n l$ (encoding a homolog of mammalian FGF) and btl (a FGF receptor homolog) specifies the tracheal branching pattern by guiding tracheal cell migration. bnl is strictly required for tracheal branching and is expressed dynamically in clusters of cells surrounding the developing tracheal system at each position where a new branch will form and grow out. In addition, localized misexpression of bnl can direct branch formation and outgrowths to new positions (Sutherland et al., 1996). We have found that the dorsal patches of $b n l$ transcript are strongly reduced or absent in punt and $t k v$ mutants, respectively, suggesting that DPP controls the region-specific activation of $b n l$ in the dorsal part of the embryo, presumably in concert with other factors. The absence of $b n l$ dorsally could be due to an indirect effect, since DPP regulates transcription of other genes in the dorsal ectoderm (see Affolter et al., 1994).

Although the absence of $b n l$ dorsal to the developing placode should by itself result in the lack of dorsal branches (as observed in $t k v$ and punt mutants), we argue (see above) that DPP signaling is also required cell-autonomously in the trachea, resulting in the region-specific activation of the gene $k n i$, for example. Indeed, the absence of kni (and its functional homolog kni-related; Rothe et al., 1992) in the developing tracheal system leads to defects closely resembling those observed in $t k v$ mutants (Chen, Kühnlein, Eulenberg, Vincent, Affolter and Schuh, unpublished). Concerning this dual role of DPP, it is worth mentioning that in the total absence of DPP, the embryo fails to build tracheal anlagen altogether and is strongly ventralized; this indicates that DPP has even earlier functions in tracheal development than those described in this work (see also de Celis et al., 1995).

\section{DPP and BNL signaling are both required for migrations along the dorsoventral axis}

Our results demonstrate that DPP signaling is required in dorsal and ventral tracheal cells for directed migration. In addition, cells which do not normally migrate towards the dorsal side of the embryo can be induced to do so upon ectopic activation of the DPP signaling pathway. This suggests that DPP signaling is required in addition to signaling via the FGF pathway for cells to move along the dorsoventral body axis. This requirement for two different signaling pathways is highlighted by the lack of dorsal migration upon ectopic expression of dominant negatively acting DPP receptors in tracheal cells; despite the presence of the bnl ligand, cells fail to move dorsally (Fig. 7). BNL as a proposed guidance cue is also ignored by nearby tracheal cells when a constitutive active DPP receptor is expressed in the trachea, with the result that cells which would migrate anteriorly start to move dorsally. Therefore, the bnl signal does not appear to be sufficient to specify the direction of primary branch outgrowth. This partial insufficiency has already been observed by Krasnow and collegues (Sutherland et al., 1996).

\section{How does DPP influence the migration behavior of tracheal cells?}

How is the dual requirement for DPP and FGF signaling molecularly integrated in tracheal cells? Although there are numerous possibilities, only two are discussed here in some detail.

In a first scenario, it is possible that DPP (maybe via KNI) activates the transcription of genes encoding receptors for yet to be identified guidance cues which help to direct tracheal cells towards defined positions. Evidence for additional factors has also been obtained in experiments in which constitutive active BTL receptor fusions were expressed during 
tracheal migration (Reichman-Fried et al., 1994), and upon ectopic expression of the BNL ligand (Sutherland et al., 1996). In addition, it has been argued that the BTL receptor is required only for the onset of tracheal migration, and not continuously throughout the migration phase (Reichman-Fried and Shilo, 1995). This raises the possibility that tracheal cells are guided by both by the spatially restricted expression of a motogen $(b n l)$ and by other guidance molecules which might be distinct for different directions of migration (e.g. anterior, dorsal, etc.).

In a second scenario, FGF and DPP signaling might be required in concert (or sequentially) for tracheal cells to become motile and follow the dynamic expression of bnl towards their target. This would imply that the motility of tracheal cells is controlled by different factors in anteriorly migrating cells (eventually by sal) and in cells migrating along the dorsoventral axis (eventually by kni). This second scenario is more difficult to reconcile with the observation that ectopic DPP signaling in the center of the placode can reprogram cells to adopt a dorsal migration behavior.

In both of these scenarios, the region-specific expression of transcription factors is instrumental for tracheal cells to adopt the proper migration behavior. The identification of the target genes of these factors should provide insight into the molecular mechanisms through which tracheal cells integrate information from their cellular environment and translate it into proper migration behaviors.

We would like to thank M. Krasnow and D. Sutherland for providing the $b n l$ cDNA before publication, B. Shilo for flies and antibodies, and K. Basler and D. Nellen for numerous fly stocks and for discussion. We are indebted to Konrad Basler for comments on the manuscript, to Shigeo Hayashi for giving us the btlGAL4 strains, and to T. Marty for the generation of the 1-eve-1, punt recombinant chromosome. We thank Michael O'Connor for hosting one of us (T. H.). The help of U. Sauder and M. Dürrenberger (from the Interdepartementale Elektronenmikroskopie, Biozentrum, Universität Basel) and Steve Therianos in sectioning and mounting Drosphila embryos was very much appreciated. Special thanks go to Rosemarie Suter and Erika Marquardt for secretarial assistance, and to Ute Nussbaumer for expert technical assistance. This work was supported by grants from the Swiss National Science Foundation and the Kanton Basel-Stadt (M. A.), the Roche Research Foundation and the European Community (E. R.), and the Graduiertenkolleg (Molekulare Genetik der Entwicklung) (C.-K. C.).

\section{REFERENCES}

Affolter, M., Nellen, D., Nussbaumer, U. and Basler, K. (1994). Multiple requirements for the receptor serine/threonine kinase thick veins reveal novel functions of $\mathrm{TGFb}$ homologs during Drosophila embryogenesis. Development 120, 3105-3117.

Ashburner, M. (1989). Drosophila: A Laboratory Manual. Cold Spring Harbor, New York: Cold Spring Harbor Laboratory Press.

Brummel, T. J., Twombly, V., Marqués, G., Wrana, J. L., Newfeld, S. J., Attisano, L., Massagué, J., O'Connor, M. B. and Gelbart, W. M. (1994). Characterization and relationship of dpp receptors encoded by saxophone and thick veins genes in Drosophila. Cell 78, 251-261.

Campos-Ortega, J. A. and Hartenstein, V. (1985). The Embryonic Development of Drosophila melanogaster. Berlin: Springer-Verlag.

Chouinard, S. and Kaufman, T. C. (1991). Control of expression of the homeotic labial (lab) locus of Drosophila melanogaster: evidence for both positive and negative autogenous regulation. Development 113, 1267-1280.

Culotti, J. G. (1994). Axon guidance mechanisms in Caenorhabditis elegans. Curr. Op. Genet. Dev. 4, 587-595. de Celis, J. F., Llimargas, M. and Casanova, J. (1995). ventral veinless, the gene encoding the Cf1a transcription factor, links positional information and cell differentiation during embryonic and imaginal development in Drosophila melanogaster. Development 121, 3405-3416.

Garriga, G. and Stern, M. J. (1994). Hams and Egls: genetic analysis of cell migration in Caenorhabditis elegans. Curr. Opin. Gen. Dev. 4, 575-580.

Glazer, L. and Shilo, B. -Z. (1991). The Drosophila FGF receptor homolog is expressed in the embryonic tracheal system and appears to be required for directed tracheal cell extension. Genes Dev. 5, 697-705.

Hartenstein, V. and Jan, Y. N. (1992). Studying Drosophila embryogenesis with P-lacZ enhancer trap lines. Roux's Arch. Dev. Biol. 201, 194-220

Hauptmann, G. and Gerster, T. (1994). 2-color whole-mount in situ hybridization to vertebrate and Drosophila embryos. Trends Genet. 10, 266.

Howard, K. (1993). Getting there? Curr. Biol. 2, 103-105.

Jürgens, G., Wieschaus, E., Nüsslein-Volhard, C. and Klunding, H. (1984). Mutations affecting the pattern of the larval cuticle in Drosophila melanogaster. II. Zygotic loci on the third chromosome. Roux's Arch. Dev. Biol. 193, 283-295.

Klämbt, C., Glazer,L. and Shilo, B. (1992). breathless, a Drosophila FGF receptor homolog, is essential for migration of tracheal and specific midline glial cells. Genes Dev. 6, 1668-1678.

Kühnlein, R. P., Frommer, G., Friedrich, M., Gonzalez-Gaitan, M., Weber, A., Wagner-Bernholz, J. F., Gehring, W. J., Jäckle, H. and Schuh, R. (1994). spalt encodes an evolutionarily conserved zinc finger protein of novel structure which provides homeotic gene function in the head and tail region of the Drosophila embryo. EMBO J. 13, 168-179.

Kühnlein, R. P and Schuh R. (1996). Dual function of the region-specific homeotic gene spalt during Drosophila tracheal system development. Development 122, 2215-2223.

Jürgens, G., Wieschaus, E., Nüsslein-Volhard, C. and Klunding, H. (1984). Mutations affecting the pattern of the larval cuticle in Drosophila melanogaster. Roux's Arch. Dev. Biol. 193, 283-295.

Lauffenburger, D. A. and Horwitz, A. F. (1996). Cell migration: A physically integrated molecular process. Cell 84, 359-369.

Lee, T., Hacohen, N., Krasnow, M. A. and Montell, D. J. (1996). Regulated Breathless receptor tyrosine kinase activity required to pattern cell migration and branching in the Drosophila tracheal system. Genes Dev. 10, 2912-2921.

Letsou, A., Arora, K., Wrana, J. L,. Simin, K., Twombly, V., Jamal, J., Staehling-Hampton, K., Hoffmann, F. M., Gelbart, W. M., Massagué, J. and O'Connor, M. B. (1995). Drosophila dpp signaling is mediated by the punt gene product: a dual ligand-binding type II receptor of the TGF $\beta$ receptor family. Cell $\mathbf{8 0}, 899-908$.

Manning, G. and Krasnow, M. A. (1993). Development of the Drosophila tracheal system. In The Development of Drosophila (ed. A. Martinez-Arias and M. Bate), Vol. 1, pp. 609-685. Cold Spring Harbor, New York: Cold Spring Harbor Laboratory Press.

Mitchison, T. J. and Cramer, L. P. (1996). Actin-based cell motility and cell locomotion. Cell 84, 371-379.

Montell, D. (1994). Moving right along: regulation of cell migration during Drosophila development. Trends Genet. 2, 59-62.

Nauber, U., Pankratz, M. J., Kienlein, A., Seifert, E., Klemm, U. and Jäckle, H. (1988). Abdominal segmentation of the Drosophila embryo requires a hormone receptor-like protein encoded by the gap gene knirps. Nature $\mathbf{3 3 6}$ 489-492.

Nellen, D., Affolter, M. and Basler, K. (1994). Receptor serine/threonine kinases implicated in the control of Drosophila body pattern by decapentaplegic. Cell 78, 225-237.

Nellen, D., Burke, R., Struhl, G. and Basler, K. (1996) Direct and long-range action of a DPP morphogen gradient. Cell 85, 357-368.

Nüsslein-Volhard, C., Wieschaus, E. and Klunding, H. (1984). Mutations affecting the pattern of the larval cuticle in Drosophila melanogaster. I. Zygotic loci on the second chromosome. Roux's Arch. Dev. Biol. 193, 267282

Penton, A., Chen, Y., Staehling-Hampton, K., Wrana, J. L., Attisano, L., Szidonia, J., Cassil, J. A., Massagué, J. and Hoffmann, F. M. (1994). Identification of two bone morphogenetic protein type I receptors in Drosophila and evidence that Brk25D is a decapentaplegic receptor. Cell 78, 239-250.

Perrimon, N., Noll, E., McCall, K. and Brand, A. (1991). Generating lineagespecific markers to study Drosophila development. Dev. Genet. 12, 238-252.

Reichman-Fried, M., Dickson, B., Hafen, E. and Shilo, B. -Z. (1994). Elucidation of the role of breathless, a Drosophila FGF receptor homolog, in tracheal cell migration. Genes Dev. 8, 428-439.

Reichman-Fried, M. and Shilo, B. -Z. (1995). Breathless, a Drosophila FGF 
receptor homolog, is required for the onset of tracheal cell migration and tracheole formation. Mech. Dev. 52. 265-273.

Rothe, M. Pehl, M., Taubert, H. and Jäckle, H. (1992). Loss of gene function through rapid mitotic cycles in the Drosophila embryo. Nature 359, 156-159.

Ruberte, E., Marty, T., Nellen, D., Affolter, M. and Basler, K. (1995). An absolute requirement for both the type II and type I receptors, punt and thick veins, for dpp signaling in vivo. Cell 80, 1889-1897.

Samakovlis, C., Hacohen, N., Manning, G., Sutherland, D., Guillemin, K. and Krasnow, M. A. (1996) Branching morphogenesis of the Drosophila tracheal system occurs by a series of morphologically distinct but genetically coupled branching events. Development 122, 1395-1407.

Shiga, Y., Tanaka-Matakatsu, M. and Hayashi, S. (1996). A nuclear GFP/ßgalactosidase fusion protein as a marker for morphogenesis in living Drosophila. Dev. Growth Differ. 38, 99-106.

Singer, M. A., Penton, A., Twombly, V., Hoffmann, F. M. and Gelbart, W. M. (1997). Signaling through both type I DPP receptors is required for anterior-posterior patterning of the entire Drosophila wing. Development 124, 79-89.

Sutherland, D., Samakovlis, C. and Krasnow, M. A. (1996). branchless encodes a Drosophila FGF homolog that controls tracheal migration and pattern of branching. Cell 87, 1091-1101.

St. Johnston, R. D., Hoffmann, F. M., Blackman, R. K., Segal, D., Grimaila, R., Padgett, R. W., Irick, H. A. and Gelbart, W. M. (1990). Molecular organization of the decapentaplegic gene in Drosophila melanogaster. Genes Dev. 4, 1114-1127.

Tautz, D. and Pfeifle, C. (1989). A non-radioactive in situ hybridization method for the localization of specific RNAs in Drosophila embryos reveals translational control of the segmentation gene hunchback. Chromosoma $\mathbf{9 8}$ $81-85$. 